\title{
Alla ricerca di nuovi significati topologici dei generi geometrico e aritmetico di una superficie algebrica.
}

\author{
Memoria di Gundo ZAPpA (a Napoli).
}

Sunto. - Si determina la struttura topologica di una superficie algebrica che ammetta una forma limite spezzata in piani nell' intorno delle rette di connessione e dei punti di connessione tripla di questa, e si inizia un ordine di ricerche che dovrà presumibilnente portare a nuovi significati topologici dei generi aritmetico e geometrico.

\section{INTRODUZIONE}

I. Il presente studio si propone di contribuire alla conoscenza dei caratteri topologici di una superficie algebrica.

Partiamo da alcune semplici osservazioni relative alle curve algebriche, osservazioni che ei hanno ispirato nello studio delle superficie.

Il genere $p$ di una curva algebrica si può, dal punto di vista topologico, interpretare, oltre che nei modi usuali, in una delle maniere seguenti:

a) Sopra la riemanniana di una curva algebrica di genere $p$, il massimo numero di cicli unidimensionali, indipendenti tra loro, e intersecantesi a due a due in zero punti, è eguale a $p$.

Infatti, se la riemanniana della curva è data da una sfera con $p$ manici, i $p$ meridiani (oppure i $p$ paralleli) dei manici soddisfano alla condizione voluta. Nè si possono trovare più di $p$ cicli ad esso soddisfacenti, altrimenti, se se ne potessero trovare, ad es., $p+1$, formata una base con detti $p+1$ cicli $e$ con altri $p-1$ cicli qualunque indipendenti tra loro e dai primi, la matrice delle intersezioni tra $\mathrm{i}$ cicli della base conterrebbe un minore d'ordine $p+1$ (quello in alto a sinistra) formato da tutti zeri, poichè, come ஓ ben noto, sulla riemanniana di una curva algebrica $(e$, più in generale, sopra una varietà bidimensionale chiusa, omogenea e unilatera) ogni ciclo unidimensionale ha intersezione nulla con se stesso. E allora la matrice delle intersezioni tra $i$ cicli della base, la quale è di ordine $2 p$, risulta a determinante nullo, poichè ogni minore d'ordine $p+1$ formato colle prime $p+1$ righe ha almeno una riga di zeri. $\mathrm{E}$ ciò non può essere.

b) Sopra la riemanniana di una curva algebrica di genere $p$, si consideri un certo numero $h>p$ di circuiti unidimensionali, intersecantisi a due a due in zero punti, e tra $\mathrm{i}$ quali ve ne siano $p$ indipendenti (cfr. $a$ )): siano essi $\Lambda_{1}, \ldots, \Lambda_{h}$. La riemanniana resterà da essi divisa in un certo numero $k$ di parti, ciascuna delle quali ha il contorno formato da un certo numero di 
cicli $\Lambda_{i}$. Supponiamo (come si può, dopo avere eventualmente aggiunto ai cicli $\Lambda_{i}$ prima scelti, altri cicli convenienti, che porremo anch'essi tra i $\Lambda_{i}$ ) che i punti situati da lati opposti di uno stesso ciolo $\Lambda$ appartengano a parti diverse. Siano $\Gamma_{1}, \ldots, \Gamma_{k}$ le parti in cui resta divisa la riemanniana. E facile vedere che è

$$
h-k=p-1
$$

Si considerino infatti in primo luogo i $p$ eicli indipendenti scelti tra gli $h$. Essi non rompono la connessione della riemanniana. La differenza tra il numero, $p$, dei cicli sin qui considerati e il numero, 1, delle parti in cui essi dividono la riemanniana è eguale a $p-1$. Si aggiunga ai suddetti $p$ cicli un nuovo ciclo scelto tra i $\Lambda_{i}$ : essso è dipendente dagli altri, valo a dire costituisce, assieme ad alcuni dei $p$ cicli anzi considerati, il contorno di una parte della riemanniana. Di conseguenza, la riemanniana resta divisa in due parti dai $p+1$ cicli sin qui considerati, onde la differenza tra il numéro, $p+1$, dei cicli e il numero, 2, delle parti in eni essi dividono la riemanniana è ancora eguale a $p-1$. Così seguitando, ogni volta che si aggiunge un nuovo ciclo, cresce di una unità anche il numero delle parti in cui resta divisa la riemanniana, onde la differenza tra questi numeri è costantemente eguale a $p-1$. E ciò avverrà anche quando saranno stati aggiunti tutti i cicli $\Lambda_{i}$, onde la (I).

Si costruisca ora nel seguente modo un complesso topologico unidimensionale, che diremo complesso associato alla riemanniana $R$ della nostra curva di genere $p$. Și faccia corrispondere a ciascuno dei cicli $\Lambda_{1}, \ldots, \Lambda_{h}$ una cella unidimensionale $\lambda_{1}, \ldots, \lambda_{h}$, e a ciascuna delle parti $\Gamma_{1}, \ldots, \Gamma_{k}$ una cella zerodimensionale $\gamma_{1}, \ldots, \gamma_{k}$, in modo tale che $\lambda_{i}$ sia incidente a $\gamma_{j}$ se e solo se $\Lambda_{i}$ fa parte del contorno di $\Gamma_{j}$. Evidentemente, ogni cella $\dot{\lambda}_{i}$ è incidente a due e due sole celle $\gamma_{j}$, mentre ogni cella $\gamma_{j} \dot{e}$ incidente ad almeno una cella $\lambda_{i}$. Otteniamo così un complesso topologico unidimensionale, $\Phi$, formato da $h$ celle unidimensionali e $k$ celle bidimensionali, e avente quindi la caratteristica di Eulero-PoIncart́ eguale a $k-h$, vale a dire, per la (I), ad $1-p$. Dalla relazione di EULkRo-PoINCARé discende allora che $p$ è $i l 1^{\circ}$ rango di connessione del coinplesso $\Phi$.

Si noti che se una curva algebrica di genere $p$ tende, variando in un sistema continuo, ad un sistema di $k$ rette, con $h$ punti di connessione (1), detta $R$ la riemanniana di una curva del sistema continuo vicina alla posi-

(1) F. SEverI, Vorlesungen über algebraische Geometrie, Leipzig, 'Teubner 1921, Anhang F. Quando una curva algebrica piana $\varphi$ d'ordine $k$ tende ad un sistema $\bar{\varphi}$ di $k$ rette distinte, tra $i$ punti comuni ciascuno a due rette di $\vec{\varphi}$ aleuni sono limiti di punti doppi di $\varphi$ e sono pa considerarsi punti doppi veri e propri di $\varphi$, mentre gli altri costituiscono singolarita che compaiono ex-novo al limite, e sono da considerarsi come virtualmente inesistenti. Essi vengono ichiamati, con Severi, punti di connessione, perchè servono a connettere tra loro le varie rette di $\bar{\varphi}$. 
zione limite, e $\vec{R}$ la riemanniana del sistema di rette, $\bar{R}$ è formata da $k$ sfere tali che due di esse hanno o no un punto in comune a seconda che le rette del sistema di piani ad essa corrispondenti hanno o no un punto di connessione in comune. Sia $P$ un punto comune a due sfere di $\bar{R}$. Quando $R$ tende ad $\bar{R}$, possiamo immaginare che essa venga ad acquistare una strozzatura che si va via via restringendo sino a ridursi al solo punto $P$.

Il cerchio di gola di questa strozzatura è un circuito unidimensionale di $R$. Otteniamo cosi, in corrispondenza agli $h$ punti di connessione, $h$ cicli unidimensionali di $R$. Essi godono delle proprietà di cui i cicli $\Lambda_{1}, \ldots, \Lambda_{h}$ sopra considerati. Infatti essi dividono la $R$ in un certo numero $k_{\text {; }}$ di parti tali che i due bordi di ogni ciclo appartengono a parti diverse (perchè, al limite, il punto $P$ cui tende il ciclo connette due sfere diverse); e inoltre, ogni altro ciclo tracciato su $R$ che non incontri quegli $h$ cicli è da essi dipendente (perchè, al limite, ogni ciclo di $\bar{R}$ che non passi per alcun punto comune a due sfere e quindi stia tutto su una sfera o è omologo a zero sulla sfera privata dei punti che la connettono alle altre, o è omologo a più cicli della sfera ciascun dei quali può ridursi mediante variazione continua ad un punto di connessione). Pertanto la considerazione della forma limite spezzata in rette ci dà un modo di suddividere la riemanniana mediante cicli unidimensionali, al modo indicato in $b$ ). Si ha inoltre che, se una curva di genere $p$ variabile in un sistema continuo tende ad un sistema di rette, tra il numero $k$ delle rette e quello $h$ dei punti di connessione passa la relazione $k-h=1-p$, che già era stata notata da Sevenr $\left(^{2}\right)$ al quale è dovato lo studio delle curve algebriche e dei sistemi continui di curve algebriche piane e sgembe mediante la considerazione di forme limite spezzate in sistemi di rette.

Fin dal 1940, Severi, intuendo l'utilità che la considerazione delle forme limiti spezzate in sistemi di spazi lineari ha nello studio delle proprietà topologiche delle varietà algebriche, mi propose di considerare il caso delle superficie $\left({ }^{3}\right)$. Ho dedicato all'argomento già vari lavori $\left({ }^{4}\right)$, alcuni dei quali sono

(2) Loe. eit. in $\left({ }^{(}\right)$.

$\left.{ }^{3}\right)$ Ved. la rubrica "Problemi, discussioni, risultati " dei "Rendiconti di Matematica e delle sue Applicazioni ", Serie V, vol, 1, p. 102, questione n. 15. Sono lieto di potere offrire, in occasione del suo $70^{\circ}$ compleanno, questo modesto frutto dei miei studi al mio Maestro Francesco Severi, cui sono profondamente grato per le direttive e i suggerimenti da lui ricevuti nelle mie ricerche, $e$ in particolari in quelle riguardanti questo argomento, negli anni in cui ho avuto la fortuna di essere suo assistente.

(4) Sulla degenerazione delle superficie algebriche in sistemi di piani distinti, con applicazioni allo studio delle rigate, "Mem. Accad. d'Italia ". 13, pp. 989-1023.

Su alcuni contributi alla conoscenza della struttura topologica delle superficie algebriche, dati dal metodo dello spezzamento in sistemi di piani, "Pont. Acad. Sci.", Acta 7, pp. 1.8

Applicazione della teoria delle matrici di Veblen e di Poincaré allo studio delle superficie spezzate in sistemi di piani, Ibidem, pp. 21.25.

Invarianti numerici di una superficie algebrica e dedusione della formula di PicardAlexander col metodo delle spezzamento in piani, * Rend. di Mat. Roma ", 5, 1946, pp. 121.130. 
note preventive e contengono risultati di cui non ho ancora dato la dimostrazione. Prima di esporre brevemente i principali punti della presente memoria, vediamo come si possa cercare di estendere alle superficie le interpretazioni $a$ ) e b) del genere di una curva. Avverrà naturalmente che, nel passaggio dalle curve alle superficie, le diverse interpretazioni di un medesimo carattere per le curve (nel nostro caso, il genere) portino a caratteri diversi per le superficie.

II. Hodge $\left({ }^{5}\right)$ ha dato per primo una interpretazione topologica del genere geometrico $p_{g}$ di una superficie, come applicazione dei suoi importanti studi sugli integrali armonici. Ricordiamo che, data una matrice quadrata simmetrica $A$ ad elementi reali, si può trovare una conveniente matrice quidrata $M$ ad elementi reali, tale che, detta $M_{-1}$ la trasposta di $M$, la matrice $M_{-1} A M$ sia una matrice diagonale.

Dicesi segnatura della $A$ la successione dei segni dei termini della $M_{-1} A M$, considerati a meno dell' ordine (legge d'inerzia).

Il risultato di HoDGE può mettersi nella forma seguente.

Si consideri, sopra la riemanniana di una superficie algebrica $F$ di genere geometrico $p_{g}$ una base $\Lambda_{1}, \ldots, \Lambda_{R_{2}}$ per i cicli bidimensionali, tale che $\Lambda_{1}$ sia il ciclo costituente una sezione piana di $F$ e sia, per ogni $i<1$ e $i \leq R_{2}$, nulla l'intersezione $\left[\Lambda_{1} \cdot \Lambda_{i}\right]$. Il numero dei termini positivi nella segnatura della matrice delle intersezioni $\left[\Lambda_{i}, \Lambda_{j}\right]\left(i=2, \ldots, R_{2} ; j=2, \ldots, R_{2}\right.$ ), (una volta scelta l'orientazione della riemanniana di $F$ in modo che sia $\left[\Lambda_{1}, \Lambda_{1}\right]>0$ ) è eguale a $2 p_{g}$.

Da questo teorema di HoDGe discende che il massimo numero $\bar{p}$ di cicli bidimensionali indipendenti della riemanniana di $F$ che abbiano ciascuno intersezione (indice di KRONECKER) nulla con sè stesso, con gli altri di essi e con il ciclo corrispondente alla sezione piana di $F$ non può superare il più piccolo dei numeri $2 p_{g}, R_{2}-2 p_{g}-1$.

Posto infatti $\left[\Lambda_{i}, \Lambda_{j}\right]=a_{i j}\left(\operatorname{con} \alpha_{i j}=a_{j i}\right)$. si consideri la quadrica

$$
\sum_{i, j=2}^{R_{2}} a_{i j} x_{i} x_{j}=0
$$

È noto che, contenendo la segnatura della matrice $\left|a_{i j}\right| 2 p_{g}$ termini positivi, ed $R_{2}-2 p_{g}-1$ termini negativi, detta quadrica contiene spazi reali di dimensione eguale al minore dei numeri $2 p_{g}-1, R_{z}-2 p_{g}-2$, ma non di dimensione superiore $\left({ }^{6}\right)$. Sia $s$ il più piccolo dei numeri $2 p_{g}, R_{2}-2 p_{g}-1$.

(5) The geometrie genus of a surface as a topological invariant, "Journ. London Math. Soc. $>, 8$, pp. 312-319.

Note on the theory of the base for curves on an algebraic surface, Journ. London Math. Soc.s, 12, pp. 58.63 .

(5) Cfr. E. Bertini, Geometria proiettiva degli iperspazi, Pisa, 1907, Cap. 6, n. 9, p. 123. 
Si prendano allora in uno, $S$, degli spazi di dimensione $s-1$ reali, $s$ punti reali indipendenti, $P_{2}, \ldots, P_{s+4}$; e siano $P_{s+2}, \ldots, P_{R_{2}}$ altri punti dell' $S_{R_{2}-2}$ ambiente che insieme ai precedenti costituiscano un sistema di $R_{2}-1$ punti reali indipendenti. Stabiliamo un nuovo sistema di coordinate $y_{2}, \ldots, y_{R_{2}}$ in modo che $P_{i}$ sia il punto in cui la $y_{i}$ vale 1 ; mentre $y_{j}$, con $j \neq i$, vale 0 $\left(i, j=2, \ldots, R_{\mathrm{q}}\right)$. Si avrà

$$
y_{i}=\sum_{j=2}^{R_{2}} \lambda_{i} x_{j} \quad\left(i=2, . ., R_{2}\right)
$$

ove le $\lambda_{i, j}$ sono reali, e il determinante $\left|\lambda_{i j}\right|$ non è nullo. Lo spazio $S$ viene ad essere ora lo spazio di equazioni $y_{s+2}=y_{s+3}=\ldots=y_{R_{1}}=0$. Trasformando la (II) mediante la (III) si otterrà l'equazione della nostra quadrica nel nuovo sistema di riferimento: sia essa

$$
\sum_{i, j=2}^{R_{2}} b_{i j} y_{i} y_{j}=0
$$

Ma poichè la quadrica contiene lo spazio $S$ che ha equazioni $y_{s+2}=\ldots=$ $=y_{R_{2}}=0$, la $(\mathrm{IV})$ è identicamente soddisfatta ponendovi $y_{s+2}=\ldots=y_{R_{2}}=0$, quindi deve aversi

$$
b_{i, j}=0 \quad(i=2, \ldots, s+1 ; j=2, \ldots, s+1) .
$$

Viceversa, se la (II) può trasformarsi, mediante convenienti equazioni (III) a coefficienti reali, nella (IV), coi coefficienti soddisfacenti alla (V), la quadrica contiene spazi reali di dimensione $s-1$, ma non superiore.

In breve, se nella matrice dei coefficienti della (II), la segnatura presenta $s$ termini di un segno ed $R_{2}-s-1$ di segno opposto, con $s \leq R_{2}-s-1$, si può eseguire sulle $x_{i}$ una conveniente sostituzione del tipo (III) a coefficienti reali, di modo che la (II) si trasformi in una equazione in cui sian nulli i coefficienti $i$ cui indici hanno ambedue valori $\leq s+1$, ma non in modo che sian nulli anche i coefficienti i cui indici hanno valore $s+2$.

Orbene, si passa dalla base $\Lambda_{2}, \ldots, \Lambda_{R_{2}}$ per $\mathrm{i}$ cicli bidimensionali della riemanniana di $F$ che hanno intersezione nulla con la sezione piana di $F$ ad un altra base per i medesimi cieli, sia $\Lambda_{2}^{\prime}, \ldots, \Lambda_{R_{a}}^{\prime}$, mediante una sostituzione

$$
\Lambda^{\prime}{ }_{i}=\sum_{j=2}^{R_{2}} \lambda_{i j} \Lambda_{j} \quad\left(i=2, \ldots, R_{2}\right)
$$

$a$ coefficienti interi, analoga alla (III), e se ̀े $a_{i j}=\left[\Lambda_{i}, \Lambda_{j}\right]$ viene ad essere $b_{i j}=\left[\Lambda_{i}^{\prime}, \Lambda_{j}^{\prime}\right]$, qualora la (IV) sia ottenuta ponendo nella (III) gli stessi coefficienti $\lambda_{i j}$ della (VI). E poichè, nelle nostre ipotesi, non è possibile far sì, con $\lambda_{i j}$ reali, che sian nulli tutti i $b_{i j}$ quando $i$ e $j$ prendono ambedue valori scelti tra $s+1$ valori prefissati, ciò non potrà farsi neanche con $\lambda_{i j}$ interi. Abbiamo allora che non è possibile passare dalla base $\Lambda_{2}, \ldots, \Lambda_{R_{2}}$ ad una base 
per cui siano nulle le reciproche insersezioni tra più di $s$ cicli. Kon sappiamo neanche se si può fare in modo che si ottenga una base per cui sono nulle le reciproche intersezioni tra $s$ cicli, poichè non sappiamo se si posson soddisfare le (V) usando equazioni (III) con $\lambda_{i j}$ interi.

Resta quindi provato che come si era affermato, il massimo numero $\mathrm{p}$ di cicli bidimensionali indipendenti della riemanniana di $\mathrm{F}$ che abbiano ciascuno intersezione nulla con sè stesso, con gli altri di essi, e col ciclo corrispondente alla sezione piana di $\mathrm{F}$, non può superare il più piccolo dei numeri $2 \mathrm{p}_{\mathrm{g}}, \mathrm{R}_{\mathrm{2}}-2 \mathrm{p}_{\mathrm{g}}-1$.

1II. Nella presente memoria pongo le premesse per la determinazione di un limite inferiore del numero $p$, per le superficie suscettibili di tendere a sistemi di piani aventi lo schema di connessione semplice già da noi considerato negli altri lavori citati: $e$ in tal modo faccio un primo passo verso la soluzione dell' analogo, per le superficie, del problema indicato per le curve in $I$, come problema $a$ ).

Contemporaneamente affronto anche l'analogo del problema b). Avevo già provato in altri lavori $\left({ }^{7}\right)$ che se una superficie $S$, variando in un sistema continuo, tende ad un sistema $M$ di piani distinti, (su eui facciamo l'jpotesi che per una retta non facente parte del limite della linea doppia non passano mai più di due piani, e per un punto non situato su tale limite non passino mai più di tre piani), detto $n$ il numero dei piani di $M, c$ il numero delle sue rette di connessione (cioè rette comuni a due piani di $M$, non facenti parte del limite della linea doppia) e $\tau$ il numero dei punti di connessione tripla di $M$ (cioè dei punti comuni a tre piani, secantisi a due a due secondo rette di connessione), il genere aritmetico $p_{a}$ di $S$ è dato dal valore

$$
p_{a}=n-c+\tau-1
$$

formula che estende la (I), ove si riguardino in essa $k$ e $h$ rispettivamente come numero delle rette e numero dei punti di connessione di un sistema di rette limite di una curva di genere $p$. Ma poichè la (I) ha significato anche indipendentemente dalla considerazione della forma limite, era naturale cercare di fare altrettanto per la (VII). A tal uopo ho dimostrato, nella prima parte di questo lavoro (n. 1,2,3) che se la superficie $S$ tende al sistema di piani $M$, si ha su $S$, in corrispondenza a ciascun punto $O$ di connessione tripla di $M$, un ciclo bidimensionale $\Lambda$ avente per limite $O$, e tale che $[\Lambda, \Lambda]=0$ su $S$. Ho provato inoltre che (n. 4, 5) la $S$ ha, nell'intorno di ciascuna retta di connessione $r$ di $M$, una varietà tridimensionale $\Gamma$, ten-

(7) Ved. i miei lavori : Su alcuni contributi..., Applicazione della teoria..., Invanianti numerici..., citati in $\left({ }^{4}\right)$. 
dente ad $r$ quando $S \rightarrow M$, e avenie il contorno costitnito dai cicli bidimensionali $A$ situati negli intorni dei punti di connessione tripla su $r$. Le varieta $\Gamma$ dividono la $S$ in $n$ parti, ciasenna delle quali ha per limite uno degli $n$ piani di $M$ (n. 6): le chiamiamo parti $V$.

In tal modo il genere aritmetico viene ad ottenersi considerando su $S$ certi cicli ciascun dei quali interseca sè stesso, ognuno degli altri, e il ciclo corrispondente alla sezione piana di $S$ in zero punti (indice di KRONEOKER): quelli che abbiam chiamato cicli $\Lambda$, e che sono in numero di $\tau$; considerando inoltre certe varietà tridimensionali (le varietà $\Gamma$ ) il cui contorno è formato dai cicli $\Lambda$, e tali che per ogni ciclo $\Lambda$ ne passan tre: il loro numero è $c$; e infine, considerando le $n$ varietà quadridimensionali in cui $S$ resta divisa dalle $\Gamma$. Il numero $n-c=\tau-1$ ci dà allora il genere aritmetico.

Si può associare (n. 7 e lavori precedenti) alla $S$ un complesso topologico bidimensionale $\Phi$, facendo corrispondere ai cicli $\Lambda$, alle varietà $\Gamma$ e alle $V$ rispettivamente celle bi-, uni- e zero-dimensionali, in modo che ogni condizione di appartenenza su $S$ si traduca in una condizione di incidenza su $\Phi$, e viceversa. E allora $p_{a}+1$ viene ad essere la caratteristica di EuLERO-PoINCARÉ del complesso $\Phi$, vale a dire $\mathrm{p}_{a}$ ci dò la differenza tra $i$ ranghi di connessione superficiale e lineare di $\Phi$.

Il complesso $\Phi$ risulta costruito in corrispondenza ad elementi dati su $S$ (varietà $V$ e $\Gamma$, e cicli $\Lambda$ ) e non in corrispondenza ad elementi di $M$ : ma l'individnazione di tali elementi si ottiene partendo da $M$ (poichè i cicli $\Lambda$ sono negli intorni dei punti di connessione tripia di $M$ eec.). Resta aperto il problema di determinare a quali condizioni debbano soddisfare dei cicli $\dot{\Lambda}^{\prime}$ bidimensionali di $\mathrm{S}$, e delle varietà $\Gamma^{\prime}$ tridimensionali di $\mathrm{S}$ aventi per contorno $\Lambda^{\prime}$, in modo che operando su di essi come si è operato sui suddetti cicli $\Lambda$ e sulle suddette varietà $\Gamma$, si ottenga un complesso topologico $\Phi^{\prime}$ per cui la ca. ratteristica di EULERO-POINOARÉ valga $\mathrm{p}_{\mathrm{a}}+1$. Solo quando si sarà data risposta a questo quesito si potrà dire risolto in pieno, per le superficie, l'analogo del problema $b$ ) indicato per le curve in $I$.

L'analogo del problema a) viene affrontato nella seconda parte del presente lavoro. Abbiam visto che due cicli $\Lambda$ distinti o coincidenti hanno tra loro intersezione nulla, e che ciascuno di essi ha anche intersezione nulla con il ciclo relativo ad una sezione piana della $S$. 亡̀ poi evidente che tale ciclo è indipendente dai cicli $\Lambda$, altrimenti avrebbe intersezione nulla con sè stesso, il che non è. Si pone allora il problema di determinare il massimo numero di cicli $\Lambda$ tra' loro indipendenti.

Introduco a tal fine il concetto di cicli $\Lambda$ pseudo-dipendenti. Chiamo precisamente pseudo-dipendenti più cicli $\Lambda$ quando costituiscono il contorno (mod. 2) di un insieme di varietà $\Gamma$. Evidentemente, eicli $\Lambda$ pseudo-dipendenti sono anche dipendenti, ma non è detto, a priori il viceversa. Più cicli non pseudo-dipendenti si diranno pseudo-indipendenti. 
Dimostro anzitutto che $i l$ massimo numero di cicli 1 pseudo-indipendenti su $\mathrm{S}$ eguaglia $i l$ secondo rango di connessione $\mathrm{r}_{2}$ del complesso $\Phi$ (nn. 8, 9).

A tal punto si pongono due domande: A) Cicli $\Lambda$ pseudo-indipendenti sono anohe indipendenti? B) Che relazione passa tra i ranghi di connessione di $\Phi$ e i caratteri invarianti di $S$ ?

Alla domanda A) va data rispoșta affermativa, ma non espongo qui la dimostrazione, perchè non la ho ancora perfezionata in tutti i suoi particolari. Comparirà in altro lavoro che farà seguito a questo.

Quanto alla domanda B) in questo lavoro mi limito a dimostrare che, per le superficie prive di coefficienti di torsione unidimensionali (e quindi anche bidimensionali) pari, $\Theta$ sotto una ipotesi che molto presumibilmente è sempre verificata (supposizione $\Sigma$ nel n. 11), il primo rango di connessione $r_{\text {, }}$ $d i \Phi$ e l'irregolarità $q$ di $\mathrm{S}$ sono legati dalla diseguaglianza $(\mathrm{n} .10,11,12$,

$$
r_{1} \leq q
$$

In particolare, per $q=0$ si ha $r_{1}=0$, e pertanto, tenuto conto della relazione, già vista, $p_{a}=r_{2}-r_{1}$, si ha che se $\mathrm{S}$ è una superficie regolare $\left(q=0, p_{\mathrm{a}}=\mathrm{p}_{\mathrm{g}}\right)$ il secondo rango di connessione $\mathrm{r}_{2}$ di $\Phi$ eguaglia il genere geometrico $\mathrm{p}_{\mathrm{g}} d i \mathrm{~S}$, e di conseguenza il massimo numero di cicli $\Lambda$ psendodipendenti eguaglia $p_{g}$.

Ho pressochè completa la dimostrazione del fatto che $r_{1}=q$, dal che segue $r_{2}=p_{g}$ in ogni caso. Conto di dare in una prossima memoria la dimostrazione di ciò.

Una volta fatto questo, e provato nel contempo che alla domanda A) va data risposta affermativa, seguirà che sopra nna superficie $S$ suscettibile di spezzarsi, al limite, in un sistema di piani, vi sono almeno $p_{g}$ cicli bidimen. sionali indipendenti, ciasoun dei quali ha intersezione nulla con se stesso, con ciasouno degli altri e con il ciclo relativo ad una sezione piana di $S$. Si avrà così un limite inferiore, $p_{g}$, per il numero $\bar{p}$ di cui al n. II di questa introduzione, il quale ammette, come s'è visto, per limite superiore il più piccolo dei numeri $2 p_{g}, R_{2}-2 p_{g}-1$.

Ho ragioni per ritenere che sia sempre $\bar{p}=2 p_{g}$, da cui, in base alla diseguaglianza $\bar{p} \leq R_{2}-2 p_{g}-1$, seguirebbe anche $R_{z} \geq 4 p_{g}+1$, diseguaglianza che vien confermata dai pochi esempi che si sanno fare.

Concludo facendo presente che le relazioni $r_{1}=q, r_{2}=p_{g}$ furono da me preannunciate in un precedente lavoro $\left(^{8}\right)$, e che tutti $\mathrm{i}$ risultati di questa memoria furono enunciati in una mia comunicazione al III. Congresso dell'Unione Matematica Italiana, tenutosi a Pisa nel settembre 1948.

(8) Applicazione della teoria ..., citato in $\left({ }^{4}\right)$. 


\section{$\S 1$. Comportamento topologico di una superficie algebrica nell'intorno delle rette di connessione e dei punti di connes- sione tripla di una sua forma limite spezzata in piani.}

1. Si consideri, in primo luogo, la superficie cubica $F$ di equazione

$$
x y z=h
$$

la quale, per $h \rightarrow 0$, tende al sistema di piani $L$ costituito dai tre piani coordinati.

Si ponga $x=\rho_{1}^{i} e^{i \varphi_{1}}, \quad y=\rho_{2} e^{i \varphi_{2}}, z=\rho_{3} e^{i \varphi_{3}}, h=r e^{i \circledast}\left(\rho_{1}, \rho_{z}, \rho_{3}\right.$ reali $\geq 0$, $\varphi_{1}, \varphi_{2}, \varphi_{3}, \Theta$ compresi tra 0 e $2 \pi$ ). Sostituendo nella (1) avremo

vale a dire

$$
\rho_{1} \rho_{2} \rho_{3} e^{i\left(\varphi_{1}+\varphi_{i}+\varphi_{3}\right)}=r e^{i \Theta}
$$

$$
\rho_{1} \rho_{2} \rho_{3}=r, \quad \varphi_{1}+\varphi_{2}+\varphi_{3} \equiv \theta
$$

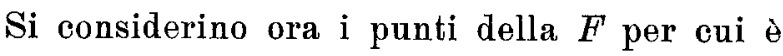

$$
\rho_{1}=\rho_{2}=\rho_{3}=r^{\frac{1}{3}} \text {. }
$$

Essi formano un ciclo bidimensionale $\Lambda$. Infatti sono in corrispondenza topologica con le coppie ordinate $\left(\varphi_{1}, \varphi_{2}\right) \quad\left(0 \leq \Theta_{1}<2 \pi ; 0 \leq \Theta_{2}<2 \pi\right)$, poichè, fissati ad arbitrio $\varphi_{1}$ e $\varphi_{2}, \varphi_{3}$ resta determinato dalla seconda delle (3), mentre $\rho_{1}, \rho_{2}, \rho_{3}$ son dati dalle (4).

Si noti che è $[\Lambda, \Lambda]=0$. Infatti ponendo, in luogo della (4),

$$
\rho_{1}=\alpha r^{\frac{1}{3}}, \quad \rho_{2}=\beta r^{\frac{1}{3}}, \quad \rho_{3}=\alpha^{-1} \beta^{-1} r^{\frac{1}{3}}
$$

( $\alpha$ e $\beta$ reali positivi fissi) si ottiene, in luogo di $\Lambda$, un ciclo $\Lambda^{\prime}$ omologo a $\Lambda$, e si ha $\left[\Lambda, \Lambda^{\prime}\right]=0$, perchè, se vi fosse un punto comune a $\Lambda$ e $\Lambda^{\prime}$, si avrebbe per la $x$ di esso, da un lato $r^{\frac{1}{3}} e^{i \phi_{1}}$, dall' altro $\alpha r^{\frac{1}{3}} e^{i \bar{\varphi}_{1}}$, vale a dire $r^{\frac{1}{3}}=\alpha r^{\frac{1}{3}}$, il che: per $\alpha \neq 1$, è impossibile.

Quando $h$ tende a zero, cioè $F$ tende ad $L$, il ciclo $\Lambda$ tende ad 0 . Infatti, se $h \rightarrow 0$, anche $r \rightarrow 0$, e pertanto anche $r^{\frac{1}{3}} \rightarrow 0$. Dalle (4) segue che $\rho_{1}, \rho_{2}, \rho_{3}$, quindi anche $x, y, z$ tendono a zero, onde $\Lambda$ tende all' origine $O$.

Concludendo sulla superficie cubica $\mathrm{xyz}=\mathrm{h}$ c'è un ciclo bidimensionale $\Lambda$ tale che $[\Lambda, \Lambda]=0$, e tendente all'origine $\mathrm{O}$ degli assi quando la superficie tende al sistema di piani $\mathrm{xyz}=0$.

2. Si consideri ora la totalità dei punti di $F$ per cui è $\rho_{2}=\rho_{3}$. Essi formano una varietà tridimensionale $\Gamma^{*}$, poichè sono in corrispondenza topologica con le terne ordinate $\left(\varphi_{1}, \varphi_{1}, \varphi_{2}\right)\left(0 \leq \rho, \leq+\infty, 0 \leq \varphi_{1}<2 \pi, 0 \leq \varphi_{2}<2 \pi\right)$. 
Il ciclo $\Lambda$, di cui al n. prec. giace su $I^{*}$, e divide quest' ultima in due parti una delle $q$ uali corrisponde ai valori di $\rho_{1}$ per cui è $\rho_{1}<\rho_{2}$ (e quindi anche $\rho_{1}<\rho_{3}$ ), l'altra, che diremo $\Gamma_{1}$, corrisponde ai valori di $\rho_{1}$ per cui è $\rho_{1}>\rho_{2}$ (e quindi anche $\left.\rho_{1}>p_{3}\right)$. Se consideriamo $\Lambda$ come facente parte di $\Gamma_{1}$, quest'ultima viene ad essere costituita dai punti di $F$ per cui è

$$
\rho_{1} \geq P_{2}=P_{3} \text {. }
$$

Il contorno di $\Gamma_{1}$ è costituito solo da $\Lambda$. Infatti, essendo i punti di $\Gamma_{\text {, }}$ in corrispondenza topologica con le terne ordinate $\left(\rho_{1}, \varphi_{1}, \varphi_{2}\right)\left(r^{\frac{1}{3}} \leq \rho_{1} \leq+\infty\right.$, $\left.0 \leq \varphi_{1}<2 \pi, \quad 0 \leq \varphi_{2}<2 \pi\right)$, il contorno di $\Gamma_{1}$ può aversi solo per $\rho_{1}=r^{\frac{1}{3}}$ o per $\rho_{1}=+\infty$. Ma per $\rho_{1}=r^{\frac{1}{3}}$ si ha appunto $\Lambda$, mentre per $\rho_{1}=+\infty$ è $\rho_{2}=\rho_{3}=0$, quindi anche $\varphi_{1}=\varphi_{2}=0$, onde si ottiene il solo punto improprio dell'asse $x$, il quale inoltre risulta un punto interno di $\Gamma_{l}$. Pertanto il contorno di $\Gamma_{1}$ è formato dal solo $\Lambda$.

Osserviamo ora, che quando $F \rightarrow L$, ciò̀ $h \rightarrow 0$, anche $r \rightarrow 0$, quindi $\rho_{2}$ e $\rho_{3}$, che sono $\leq r^{\frac{1}{3}}$, tendono a zero. Di conseguenza $y$ e $z$ tendono a zero, onde $\Gamma_{1}$ tende all' asse delle $x$.

In modo analogo, ponendo $\rho_{1}=\rho_{3}$, e rispettivamente $\rho_{1}=\rho_{2}$, si costruisce su $F$ una varieta tridimensionale $\Gamma_{2}$, e rispettivamente $\Gamma_{3}$, aventi ambedue per contorno $\Lambda$, e tendenti la prima all'asse delle $y$, l'altra all'asse delle $z$ quando $F$ tende ad $L$.

Concladendo, sulla superficie cubica $\mathrm{xyz}=\mathrm{h}$ ci sono tre varietà tridimensionali $\Gamma_{1}, \Gamma_{2}, \Gamma_{3}$, aventi ciascuna per contorno il ciclo $\Lambda$ di cui al $n$. prec., e tendenti ciascuna ad uno degli assi coordinati quando la superficie tende al sistema di piani $\mathrm{xyz}=0$.

3. Pel sistema di piani $L$ limite della superficie $F(x y z)=h$ di cui ai nn. prec., il punto $O$ è un punto di connessione tripla, mentre i tre assi coordinati sono rette di connessione. Si consideri ora una generica superficie algebrica $S$, d'ordine $n$, che ammetta come limite un sistema di piani $M$, di cui faccian parte $\mathrm{i}$ piani $x=0, y=0, z=0$, in guisa tale che $\mathrm{i}$ tre assi risultino rette di connessione, e il punto $O$ punto di connessione tripla.

Il sistema $M$ è costituito dal sistema $L$ più un ulteriore sistema $N$ di $n-3$ piani. Si consideri una posizione di $S$ vicina ad $M$, e una posizione di $F$ vicina ad $L$. Si proietti il ciclo $\Lambda$, di cui al $n .1$, da $O$. Si otterrà un sistema $\infty^{2}$ di rette di equazioni

$$
x=m y=n z
$$

ove $m$ ed $n$ sono, numeri complessi tali che un punto di coordinate $x_{0}, \frac{1}{m} x_{0}, \frac{1}{n} x_{0}$ (con $x_{0}$ conveniente) appartenga ad $\Lambda$; perchè ciò sia, in base 
alle (4), occorre e basta che $m$ ed $n$ abbiano ambedue modulo 1. Ma allora tutti i punti della retta (6) hanno le tre coordinate di egual modulo, e pertanto le tre intersezioni della (6) con la $F$ soddisfano tutte e tre alle (4), vale a dire appartengono a $\Lambda$.

Si noti che la (6) (postovi $|m|=|n|=1$ ) ha le tre intersezioni con $F$ sempre tra loro distinte, e quindi il ciclo $\Lambda$ è proiettato da 0 triplamente, senza punti di diramazione. Sia $K$ il cono proiettante $\Lambda$ da $O$.

Ciaseuna retta di $K$ incontra la $S$ in $n$ punti, tre dei quali tendono ad $O$ quando $S$ tende ad $M$ (perchè $O$ è punto triplo per $M$ ). Tali tre punti, al variare della retta in $K$, costituiscono una varietà $\bar{\Lambda}$ a due dimensioni. Si vede facilmente che $\bar{\Lambda}$ non ha contorni. Infatti, si noti anzitutto che le tre intersezioni vicine ad $O$ che una retta di $K$ ha con $S$ son sempre distinte, se la $S$ è vicina ad $M$, poichè in tal caso le tangenti condotte da $O$ alla $S$ con punto di contatto vicino ad $O$ sono vicine ai piani coordinati $x=0, y=0$, $z=0$, mentre le rette di $K$ non sono vicine a questi piani.

Ne segue che la corrispondenza $(3,1)$ tra $i$ punti di $\bar{\Lambda}$ e le rette di $\mathrm{K}$ non possiede diramazioni. Pertanto preso un punto $\bar{P}$ di $\bar{\Lambda}$ e la retta $r=O \bar{P}$ di $K$, la corrispondenza tra i punti di $\bar{\Lambda}$ situati nell'intorno di $\bar{P}$ e le rette di $K$ situate nell'intorno di $r$ o biunivoca e continua. Analogamente, poichè anche la corrispondenza tra le rette di $K$ e i panti di $\Lambda$ non possiede diramazioni, tra le rette di $K$ situate nell'intorno di $r$ e i punti di $\Lambda$ situati nell' intorno di uno, sia esso $P$, dei tre punti comuni ad $r$ e $\Lambda$, intercorre una corrispondenza biunivoca e continua. Di conseguenza è biunivoea e continua anche la corrispondenza tra l'intorno di $\bar{P}$ e quello di $P$, e poichè, non avendo $\Lambda$ contorni, l'intorno di $P$ è sempre omeomorfo ad una bicella, anche l'intorno di $\bar{P}$ lo è sempre, comunque si scelga $\bar{P}$ su $\bar{\Lambda}$. E quindi $\bar{\Lambda}$ è un ciclo, al pari di $\Lambda$.

É evidente poi che, quando $\mathrm{S}$ tende ad $\mathrm{M}$, il ciclo $\bar{\Lambda}$ tende al punto 0 , poichè $\bar{\Lambda}$ è formato per l'appunto da quelle tra le intersezioni delle rette di $K$ con $S$, che tendono ad $O$ per $S \rightarrow M$.

Si noti infine che è su $\mathrm{S},[\bar{\Lambda}, \bar{\Lambda}]=0$. Basta a tal fine, sostituire alla (4) le $\left(4^{\prime}\right)$, ove si sian presi $\alpha$ e $\beta$ diversi da 1 . Il ciclo $\Lambda$ viene sostituito come risulta dal n. 1 , da un ciclo $\Lambda^{\prime}$ tale che $\left[\Lambda, \Lambda^{\prime}\right]=0$; il cono $K$ viene sostituito da nn cono $K^{\prime}$ non avente rette a comune con $K$ (altrimenti $\Lambda$ avrebbe punti a comune con $\Lambda^{\prime}$ ); e infine il ciclo $\bar{\Lambda}$ viene sostituito da un ciclo $\bar{\Lambda}^{\prime}$ non avente punti a comune con $\bar{\Lambda}$ (altrimenti $K$ avrebbe rette a comune con $K^{\prime}$ ). Ne segue che è $\left[\bar{\Lambda}, \bar{\Lambda}^{\prime}\right]=0$, e poichè $\Lambda^{\prime}$ è omotopo, e quindi anche omologo a $\bar{\Lambda}$, si ha $\left[\bar{\Lambda}, \bar{\Lambda}^{\prime}\right]=0$, come si era detto.

Si ha pertanto che:

Se la superficie algebrica $\mathrm{S}$ tende al sistema di piani $\mathrm{M}$, la $\mathrm{S}$ possiede, in corrispondenza ad ogni punto, $\mathrm{O}$, di connessione tripla di $\mathrm{M}$, un ciclo bidi. mensionale $\bar{\Lambda}$, che ha per limite $\mathrm{O}$, e tale che $[\bar{\Lambda}, \bar{\Lambda}]=0 \mathrm{su} \mathrm{S}$. 
4. Si proiettino ora da $O$ su $S$ i punti di $\Gamma$ situati nell'intorno di $\Lambda$. Una retta $r$ uscente da $O$ passante per un punto di $I^{\prime}$ deve potersi rappre. sentare nella forma (6) con $|m|=|n| \geq 1$. Ma allora per tutti i punti

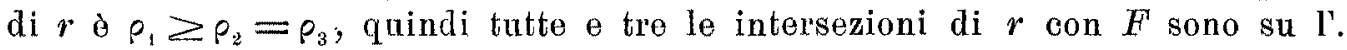
La $r$ ha tre intersezioni con $S$ che tendono ad $O$ quando $S$ tende ad $M$. La totalità di tali terne di intersezioni costituiscono una varietà a tridimensioni $\bar{\Gamma}$, definita però ora unicamente nelle vicinanze di $\bar{\Lambda}$, e che poi prolungheremo opportunamente: Ragionando in modo analogo a quello tenuto nel $\mathrm{n}$. prec., si prova che $\vec{\Gamma}$ ha per contorno $\vec{\Lambda}$, oltre all'omologo del contorno che abbiamo imposto per delimitare l'intorno di $\Gamma$ che abbiamo proiettato da 0 .

Si sechino tanto $F$ che $S$ con un piano $x=c$, con $|c|>r^{\frac{1}{3}}$ e vicino ad $r^{\frac{1}{3}}$ medesimo. La $F$ è secata secondo una curva $D$ la quale, quando $F \rightarrow L$, tende ad un sistema di tre rette avente un punto doppio nell'intersezione $A$ del piano con l'asse $x$; mentre $\Gamma$ è secata secondo un ciclo lineare $\gamma$ che, per $F \rightarrow L$, tende ad $A$. La riemanniana della $D$ (che indicheremo ancora con $D$ ) nell'intorno di $A$ ha la forma di un tratto di tubo, il quale. al tendere di $F$ ad $L$, subisce al centro una strozzatura sempre più marcata; al limite, il tubo si riduce a due calotte tangenti, e la strozzatura si riducé al punto di contatto $A$ (fig. 1).

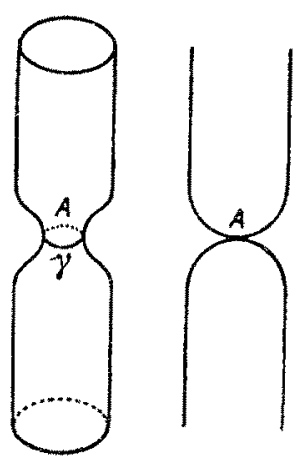

Fig. 1.

Il ciclo $\gamma$ lineare non e altro the un ciclo analogo al circolo di gola della strozzatura, come si potrebbe anche vedere ricorrendo alle formule dei $\mathrm{nn}$. prec.

La $S$ è secata dal piano $x=c$ secondo una curva $E$, ohe, quando $S \rightarrow M$, tende ad un sistema di rette avente un punto doppio in $A$; mentre $\overline{\mathbf{\Gamma}}$ è secata secondo un ciclo lineare $\bar{\gamma}$ che, per $S \rightarrow M$, tende ad $A$. La riemanniana della $E$ (che diremo ancora $E$ ) nell'intorno di $A$ ha forma analoga a quella della $D$ (fig. 1) e si comporta analogamente passando al limite. Il ciolo $\bar{\gamma}$, dovendo essere un ciclo di $E$, non omologo a zero nell' intorno di $A$, (altrimenti anche $\gamma$ lo sarebbe) e riducentesi ad $A$ per $S \rightarrow M I$, deve essere omologo al oircolo di gola della strozzatura di $E$, o ad un suo maltiplo. Ma se immagi- 
niamo che $S$ tenda ad una superficie spezzata in $F$ e in una ulteriore parte, $E$ tende ad una curva spezzata in $D$ e in un ulteriore parte, e $\bar{\gamma}$ tende a $\gamma$. E poichè $\gamma$ è omologo al circolo di gola della strozzatura di $D, \vec{\gamma}$ deve essere omologo al circolo di gola della strozzatura di $E$, e non ad un suo multiplo.

Supponiamo ora che la $M$ abbia sull'arse delle $x$ qualche altro punto di connessione tripla oltre $O$, e sia $O$, uno di essi. Allora la $S$ ha, nell' intorno di $O$, un eiclo bidimensionale godente di proprietà analoghe a quelle di $\bar{\Lambda}$, e

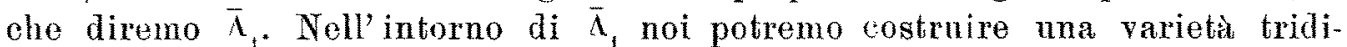
mensionale analoga alla $\bar{\Gamma}$, che diremo $\bar{\Gamma}_{1}$, avente per contorno $\bar{\Lambda}_{1}$. La $\bar{\Gamma}_{1}$ es secata da un piano $x=$ cost non troppo vicino ad $O_{1}$, nè troppo lontano, secondo un cielo $\bar{\gamma}_{1}$ il quale è omologo al cerchio di gola della strozzatura presentata dalla curva $E_{1}$, sezione di $S$ col piano $x=$ cost, nell'intorno del punto in cui questo piano incontra l'asse $x$. Potremo ora prolungare la $I$ e la $\bar{\Gamma}_{1}$ fuori dagli originari campi di definizione, facendo corrispondere, con una opportuna legge, ad ogni valore $\bar{x}$ di $x$ non troppo vicino a zero, o alla ascissa di $O_{1}$, un ciclo $\gamma_{0}$ omologo al cerchio di gola della strozzatura della curva sezione di $S$ col piano $x=\bar{x}$; e ciò potrà farsi in modo che, in corrispondenza ai valori $\bar{x}$ per eni la $\bar{\Gamma}$, o la $\bar{\Gamma}_{1}$, siano già definite, il ciclo $\bar{\gamma}_{0}$ coincida col ciclo $\bar{\gamma}$, o rispettivamente col ciclo $\bar{\gamma}_{1}$, di cui sopra. In tal modo, si viene a costruire una varietà tridimensionale, che diremo, in breve $\bar{\Gamma}_{0}$, luogo dei cicli $\bar{\gamma}_{0}$, e avente pér contorno il ciclo $\bar{\Lambda}$, il ciclo $\bar{\Lambda}_{1}$, e gli altri cicli bidimensionali definiti in modo analogo nell'intorno di ciascun altro eventuale punto di connessione tripla di $S$ situato sull'asse $x$. E poi evidente che quando $S$ tende ad $M, \bar{\Gamma}_{0}$ tende all' asse $x$, perchè $\bar{\gamma}_{0}$ tende ad un punto dell' asse $x$.

Si ha pertanto che:

Se la superficie algebrica $\mathrm{S}$ tende al sistema di piani $\mathrm{M}$, la $\mathrm{S}$ possiede, nell' intorno di ciascuna retta di connessione di $\mathrm{H}$, una varietc̀ tridimensionale $\overline{\mathrm{T}}$, la quale tende alla retta di connessione quando $\mathrm{S} \rightarrow \mathrm{M}$, e ha per contorno $i$ cicli $\bar{\Lambda}$ (n. prec.) situati negli intorni dei punti di connessione tripla appar. tenenti alla retta di connessione considerata.

5. Determiniamo ora l'interzezione di due diverse varietà $\bar{\Gamma}$, siano esse $\tilde{\Gamma}_{1}$ e $\bar{\Gamma}_{z}$. Chiamiamo $r_{1}$ ed $r_{2}$ le rette di connessione di $M$, nel oui intorno esse si trovano. Posson darsi tre casi:

a) Le rette $r_{i}$ ed $r_{z}$ non appartengono al medesimo piano di $M$. Allora, evidentemente, $\vec{\Gamma}_{1}$ e $\bar{\Gamma}_{2}$ non hanno punti a comune.

b) Le rette $r_{1}$ ed $r_{2}$ si incontrano in un punto di connessione tripla di $M$. Allora, in base ai nn. pree., $\Gamma_{1}$ e $\overline{\Gamma_{2}}$ hanno a comune un ciclo bidimensionale $\Lambda$, situato nell' intorno del punto di connessione tripla.

c) Le rette $r_{1}$ ed $r_{2}$ si incontrano in un punto cuspidale. Mostreremo che in tal caso $\bar{\Gamma}_{1}$ e $\bar{\Gamma}_{\mathrm{z}}$ hanno intersezione nulla. 
Consideriamo, come al solito, in primo luogo un caso particolare. Sia lata la rigata cubica $F$ di equazione

$$
z\left(a x^{2}+b y^{2}+x y\right)=c x y
$$

avente come direttrice doppia l'asse z. Essa tende, quando $a, b, c$ tendono a zero, al sistema dei piani $L$ di equazione $x y z=0$.

Supponiamo che $a, b$, siano funzioni continue di un parametro $t$, tendenti a zero per $t \rightarrow t_{0}$. In tal caso $F$ descrive, nel tendere ad $M$, un sistema $\infty^{\prime}$ di superficie.

Il fascio delle generatrici di $F$ tende allora $\left(^{9}\right)$ ad un sistema di tre fasci di rette: uno situato nel piano $y=0$ e avente il centro in un punto $P$ della retta di connessione $y=0, z=0$; il secondo sitnato nel piano $z=0$ e arente il centro nella origine $O$; il terzo situato nel piano $x=0$ e avente il centro in un punto $Q$ della retta di connessione $x=0, z=0$.

La $F$.è secata da un piano $x=$ cost secondo una cubica razionale $G$ che, al tendere di $F$ ad $M$, tende ad un sistema di tre rette presentante un punto di connessione nel punto $A$ comune all' asse $x$ e al piano $x=$ cost. La varietà tridimensionale $\Gamma_{1}$ che, ginsta il n. prec., è su $F$ nell'intorno della retta di connessione $y=z=0$, è secata dal piano $x=$ cost, secondo un ciclo unidimensionale $\gamma$ giacente su $C$, $e$ tendente ad $A$ quando $F \rightarrow L$. Supponiamo che $A$ sia distinto da $P$.

Per ciascan punto di $\gamma$ passa una generatrice di $F$. La totalità di queste generatrici dà luogo ad una varietà tridimensionale $\Gamma^{*}$, la quale risulta omologa a $\Gamma_{1}$. Infatti secando $\Gamma$ e $\Gamma_{1}^{*}$ mediante un piano $x=\bar{x}$, diverso dal piano $x=$ cost di cui sopra, si ottengono due cicli $\delta$ e $\delta^{*}$, ambedue $\mathrm{i}$ quali sono omologhi al circolo di gola della strozzatura presentata dalla curva $D$ comune a $x=\bar{x}$ e ad $F$, nell' intorno del punto $B$ comune a $x=\bar{x}$ e all' asse $x$. Quando $F$ tende ad $L$, e quindi $\gamma$ ad $A$, le generatrici pei punti di $\gamma$ tendon tutte all' asse $x$, perche $A$ è distinto da $P$ e pertanto l'unica generatrice-limite passante per $A$ ̀̀ l'asse $x$. La varietà $\Gamma_{1}^{*}$ può quindi essere sostituita alla, $\Gamma_{1}$, poichè soddisfa alle proprietà delle $\bar{\Gamma}$ del $\mathrm{n}$. prec.

In modo analogo possiamo costruire una varietà tridimensionale $\Gamma_{2}^{*}$ neíl'intorno della retta di connessione $x=0, z=0$.

Le due varietà $\Gamma_{1}^{*}$ e $\Gamma_{2}^{*}$ non hanno punti a comune. Infatti esse sono formate da generatrici di $F$, tra loro distinte; e poichè due generatrici distinte su $F$ hanno intersezione nulla, altrettanto avviene per $\Gamma_{1}^{*}$ e $\Gamma_{z}^{*}$.

Si abbia ora una superficie algebrica $S$ tendente ad un sistema di piani $M$. Sia $X$ un punto cuspidale di $M$; possiamo supporre che $X$ appartenga anche ad $S$, e sia un punto della linea doppia di $S$. Infatti $X$, essendo un punto cuspidale, giace sulla linea doppia di $M$, cioè è limite di un punto $X^{\prime}$ della

(9) Op. Sulla degenerazione..., eit. in (4), p. 1006. 
linea doppia di $S$ : basta allora fare eseguire ad $S$ una traslazione che porti $X^{\prime}$ in $X$, e si otterrà una nuova superficie $S^{\prime}$, che tende ad $M$ anche essa al tendere di $S$ ad $M$.

Possiamo inoltre supporre che $X$ cada nell' origine $Q$, che la retta doppia di $M$ cui $X$ appartiene sia l'asse $z$, e che le rette di connessione di $M$ per $X$ siano gli assi $x$ e $y$. Si può allora eseguire, nell' intorno di $O$, una proiezione dei punti di $F$ su $S$, facendo corrispondere a ciaseun punto $T$ di $F$ quello, tra le intersenioni di $O T$ con $S$, che tende ad $O$ per $S \rightarrow M$. Le varietà $\Gamma_{1}^{*}$ e $\Gamma_{2}^{*}$ vengono proiettate in due varietì che, almeno nell'intorno di $O$, posson considerarsi come varietà $\bar{\Gamma}$ soddisfacenti alle proprietà del $n$. prec. (efr. ragionamenti dei nn. 3 e 4) e che non hanno punti a comune. E ciò ¿̀ sufficiente per provare che due varieta $\dot{\Gamma}$, situate nell' intorno di due rette di connessione $r_{1}$ ed $r_{2}$ passanti per lo stesso punto cuspidale di $M$, hanno intersezione nulla.

Concludendo:

$L^{\prime}$ intersezione di due distinte varietà $\bar{\Gamma}$ di $\mathrm{S}$ è un ciclo $\bar{\Lambda}$ se le due varietà $\bar{\Gamma}$ sono nell'intorno di due rette di connessione di $\mathrm{M}$ convergenti in un punto di connessione tripla; in ogni altro caso l' intersezione di due distinte varietà $\bar{\Gamma} \grave{e ̀ ~ n u l l a . ~}$

6. Mostreremo ora che, se $n$ è l'ordine della $S$, questa resta divisa dalle varietà $\bar{\Gamma}$ in $n$ varietà quadrimensionali, ciascuna delle quali ha il contorno formato da varietà $\bar{\Gamma}$.

Si prendano su $M$ due punti $A$ e $B$ appartenenti il primo al piano $\alpha_{i}$, l'altro al piano $\alpha_{h}$ diverso da $\alpha_{i}$. Una linea $\sigma$ tracciata su $M$ e congiungente $A$ e $B$ deve necessariamente incontrare una delle rette di connessione giacenti su $\alpha_{i}$ : sia essa $r$, e sia $\alpha_{j}$ l'altro piano di $M$ che passa per $r$, oltre $\alpha_{i}$. Sia poi $X$ il punto comune a $\sigma$ ed $r$, e siano $Y$ e $Z$ due punti, sitnati su $\sigma$, vicini ad $X$, e situati da bande opposte rispetto ad esso, vale a dire uno su $\alpha_{i}$, l'altro su $\alpha_{j}$.

Siano ora $\bar{A}$ e $\bar{B}$ due punti di $S$ aventi come punti limiti, per $S \rightarrow M$, rispettivamente $A$ e $B$, e sia $\bar{\sigma}$ una linea conginngente $\bar{A}$ e $\bar{B}$, avente per limite $\sigma$. Siano poi $\bar{Y}$ e $\bar{Z}$ due punti aventi per limiti $Y$ e $Z$.

La varietà $\vec{\Gamma}$ avente per limite $r$ divide i punti di $S$ situati nel suo intorno in due classi, e non si può passare, rimanendo in detto intorno, da una classe all' altra senza attraversare $\bar{\Gamma}$. I punti della prima classe hanno per limite punti di $\alpha_{i}$, quelli della seconda classe punti di $\alpha_{j}$; e poichè $Y$ è su $\alpha_{i}$, e $Z$ su $\alpha_{j}$, si ha che $\bar{Y}$ e $\bar{Z}$ appartengono a classi diverse, e quindi esiste su $\bar{\sigma}$ un punto $\bar{X}$, situato tra $\bar{Y}$ e $\bar{Z}$, avente per limite $X$, e appartenente a $\bar{\Gamma}$.

Di qui discende che per andare, su $S$, da un punto $\bar{A}$ tendente ad un punto $A$ del piano $\alpha_{i}$ di $M$, ad un punto $\bar{B}$ tendente ad un punto $B$ di un altro piano di $M$ occorre attraversare una delle variéă $\overline{\mathrm{T}}$ aventi per limite rette di connessione di $M$ appartenenti ad $\alpha_{i}$. Pertanto i punti di $S$, quale $\bar{A}$, 
che tendono a punti di $\alpha_{i}$, formano nna varietì quadrimensionale avente il contorno costituito dalle varietat $\overline{\mathrm{T}}$ che tendono a rette di connossione di $M$ giacenti in $\alpha_{i}$.

Riassumendo le cose più importanti sin qui dette, possiamo concludere così:

Se la superficie algebrica $\mathrm{S}$ ha per limite il sistema di piani $\mathrm{M}$, la $\mathrm{S}$ ha, in corrispondenza a ciascuno dei $\tau$ punti di connessione tripla di $\mathrm{M}$, un ciclo bidimensionale $\bar{\Lambda}$ tendente al punto di connessione tripla quando $\mathrm{S} \rightarrow \mathrm{M}$, e tale che $[\bar{\Lambda}, \bar{\Lambda}]=0$. Detti $\bar{\Lambda}_{1}, \bar{\Lambda}_{2}$ due distinti di tali cicli $\bar{\Lambda}$, si ha $\left[\bar{\Lambda}_{1}, \bar{\Lambda}_{2}\right]=0$.

La $\mathrm{S}$ ha inoltre, in corrispondenza a ciascuna delle c relte di.connessione di $\mathrm{M}$, una varietà tridimensionale $\overline{\mathrm{T}}$, avente il contorno costituito dai cicli $\bar{\Lambda}$ situati negli intorni dei punti di connessione tripla appartenenti alla retta $d i$ connessione, e tendente alla retta di connessione stessa quando $\mathrm{S} \rightarrow \mathrm{M}$. Due varietà $\bar{\Gamma}$ hanno a comune un ciclo $\bar{\Lambda}$ se le corrispondenti rette di connessione hanno a comune un punto di connessione tripla; altrimenti hanno intersezione nulla.

Se $\mathrm{n}$ è.l'ordine di $\mathrm{S}$, questa resta divisa dalle varietò $\bar{\Gamma}$ in $\mathrm{n}$ varietà quadrimensionali $\overline{\mathrm{V}}$, ciascuna delle quali tende ad uno degli $\mathrm{n}$ piani di $\mathrm{M}$ quando $\mathrm{S} \rightarrow \mathrm{M}$, ed ha il contorno formato dalle varielà $\overline{\mathrm{T}}$ che tendono a rette $d i$ connessione del suddetto piano. Due varietà $\overline{\mathrm{V}}$ hanno a comune una varietà $\overline{\mathrm{T}}$ se $i$ piani di M a cui esse tendono hanno a comune una retta di connessione; altrimenti non hanno punti a comune.

D' ora in poi, non essendoci più timore di ambiguità, indicheremo con $\Lambda, \Gamma$ e $V$ le varietà $\sin q u i$ indicate con $\bar{\Lambda}, \bar{r}, \bar{V}$, onde chiameremo $\Lambda_{1}, \Lambda_{2}, \ldots, \Lambda_{\tau}$ i $\tau$ cicli bidimensionali $\Lambda$, diremo $\Gamma_{1}, \Gamma_{2}, \ldots, \Gamma_{c}$ le $c$ varietà tridimensionali $\Gamma$, e indicheremo con $\Lambda_{1}, \Lambda_{2}, \ldots, \Lambda_{n}$ le $n$ varietà quadrimensionali $V$.

\section{§ 2. Diseguaglianze tra i caratteri topologici di una superficie algebrica e quelli del complesso bidimensionale associato.}

7. E noto (10) che, detto $p_{a}$ il genere aritmetico di $S$, e $\tau, c, n$, rispettivamente il numero dei punti di connessione tripla, delle rette di connessione, e dei piani del sistema $M$ limite di $S$, o, il che è la stessa cosa, rispettivamente il numero dei cicli bidimensionali $\Lambda$, delle varietà tridimensionali $\Gamma$ e delle varietè quadrimensionali $V$ di $S$, si ha

$$
p_{a}=n-c+\tau-1 \text {. }
$$

In un lavoro precedente $\left({ }^{11}\right)$ ho anche dimostrato che si pui associare al sistema di piani $M$ limite di $S$ un complesso topologico bidimensionale $\Phi$, facendo corrispondere ad ogni punto di connessione tripla di $M$ una cella bidimensionale di $\Phi$, ad ogni retta di connessione di $M$ una cella unidimensionale di $\Phi$, e ad ogni piano di $M$ una cella a zero dimensioni di $\Phi$, in modo

(10) Ved. formula (VII) dell'Introduzione a questo lavoro.

(ii) Applicazione della teoria..., cit. in ( ${ }^{(4)}$. 
the due celle di $\Phi$ le eai dimensioni differiscono di ana unitia siano incidenti se e solo se i corrispondenti elementi di $M$ si appartengono.

Pertanto $\Phi$ ha $\tau$ colle bidimensionali, $c$ celle unidimensionali ed $n$ cello zerodimensionali Detti $r_{9}, r_{t}, r_{2}$ i ranghi di connessione di dimensione rispettivamente 0,1 e $2 \mathrm{di} \Phi$, si ha, dalla formula di Eulero-Porncaré applicata a $\Phi$,

$$
n-c+\tau=r_{0}-r_{1}+r_{2}
$$

ossia, visto che $\Phi$ (al pari di $M$ ) è connesso, e quindi $r_{0}=1$,

$$
n-c+\tau-1=r_{2}-r_{1} \text {. }
$$

Dalla (7) e dalla (8) segue

vale a dire, chiamando $\Phi$ complesso associato al sistema M :

Se la superficie algebrica $\mathrm{S}$ tende al sistema di piani M., il genere aritmetico di $\mathrm{S}$ eguaglia la differenza tra $i$ ranghi di connessione superficiale e lineare del complesso topologico $\Phi$ associato ad Mr.

8. Diamo ora un'altra interpretazione, utile per il seguito, al numero $r_{2}$.

Si consideri un complesso topologico bidimensionale $\Phi$, e un suo sot. tocomplesso unidimensionale $K$. Diremo anticontorno di $K$ la somma (mod. 2) delle celle bidimensionali di $\Phi$ ciascuna delle quali $\dot{\theta}$ incidente a qualch $\theta$ cella unidimensionale di $K$, ed è contata tante volte quante sono le celle unidimensionali di $K$ cui è incidente.

Sia $\mid \eta_{i k} \cdot\left(i=1, \ldots, \alpha_{1}, k=1, \ldots, \alpha_{2}\right.$, essendo $\alpha_{1}$ ed $\alpha_{2}$ rispettivamente il numero delle celle unidimensionali, e quello delle celle bidimensionali di $\Phi$ ) la seconda matrice di incidenza di $\Phi$, e sia $\left|x_{1}, \ldots, x_{a_{1}}\right|$ il simbolo del sottocomplesso $K$. 亡̀ evidente che il simbolo dell'anticontorno $\mathrm{A} d i \mathrm{~K}$ è dato dal prodolto tra matrici (12)

$$
\left|x_{i}\right| \cdot\left|\eta_{i k}\right|
$$

Basta, a prova di ciò, ragionare in modo analogo a quello che ordinariamente conduce alla determinazione del contorno di un sottocomplesso (non orientato).

Più celle bidimensionali di $\Phi$ si diranno dipendenti se esiste un sottocomplesso unidimensionale il cui anticontorno è costituito da tutte, o da parte delle celle medesime; indipendenti nel caso contrario.

Vogliamo dimostrare che:

Il secondo rango di connessione $\mathrm{r}_{2} d i \Phi$ eguaglia $i l$ massimo numero di celle bidimensionali indipendenti di $\Phi$.

È noto $\left({ }^{13}\right)$ che, detta $\rho_{2}$ la caratteristica della matrice $\left|\eta_{i k}\right|$, si ha

$$
\rho_{2}=\alpha_{2}-r_{2}
$$

(12) Per le nozioni di topologìa combinatoria qui richiamate cfr. SEveri, Conferenza di Geometria Algebrica, Bologna, Zanichelli, 1930 oppure Severi. Topologia, Buenos Aires, 1931.

(13) Op. eit. in $\left({ }^{12}\right)$. 
Sia $r_{z}$ il massimo numero di celle bidimensionali índipendenti di $\Phi$. Mustriamo anzitutto che è $r_{2} \geq r_{z}$.

La matrice $\eta_{i k}$ ha $\rho_{2}$ righe indipendenti. Supponiano, per fissare le idee, che tali siano le prime $\rho_{2}$ righe. Si consideri allora il sistema di equazioni

$$
\begin{aligned}
& \eta_{1,1} x_{1}+\ldots+\eta_{\alpha_{1}, 1} x_{\alpha_{1}}=0 \\
& \ldots \ldots \ldots \ldots \\
& \ldots \ldots \ldots \ldots \\
& \eta_{1, \rho_{2}} x_{1}+\ldots+\eta_{x_{1}, \rho_{2}} x_{\alpha_{1}}=0 \\
& \eta_{1, \rho_{2}+1} x_{1}+\ldots+\eta_{x_{1}, p_{2}+1} x_{\alpha_{1}}=1 \\
& \ldots \ldots \ldots \ldots \\
& \ldots \ldots \ldots \ldots \\
& \ldots \ldots \ldots \ldots \\
& \eta_{1, \alpha_{2}} x_{1}+\ldots+\eta_{\alpha_{1}, \alpha_{2}} x_{\alpha_{1}}=1
\end{aligned}
$$

La matrice incompleta di tale sistema, cioè la $\left|\eta_{i k}\right|$ ha caratteristica $\rho_{2}$, mentre la matrice completa ha caratteristica $\rho_{2}+1$ (infatti orlando un determinante non nullo d'ordine $\rho_{2}$ estratto dalle prime $\rho_{2}$ xighe di $\left|\eta_{i k}\right|$ si ha un determinante non nullo della matrice completa). Quindi il sistema non ha soluzioni. Se in luogo del sistema anzidetto se ne considera un altro che differisca da esso perchè $i$ secondi membri delle ultime $\alpha_{2}-\rho_{2}$ equazioni anzichè essere tutti eguali ad 1, sono in parte eguali a zero, in parte eguali ad 1, senza ohe però sian tutti nulli, egualmente (come si vede in modo analogo al caso precedente) il sistema non ha soluzioni.

Ciò equivale a dire che un complesso bidimensionale $A$ il cui simbolo ha $\mathrm{i}$ primi $\rho_{2}$ elementi eguali a zero, e i rimanenti non tutti nulli non può essere anticontorno di un complesso unidimensionale $K$ di $\Phi$, altrimenti il sistema ottenuto dal sistema (12) ponendo, a secondo membro, i termini del simbolo di $A$, ammetterebbe come solazione il simbolo di $K$, mentre tale sistema, in base a quanto abbiamo visto, non ha soluzioni.

Tenuto conto della (11) possiamo quindi concludere che le ultime $r_{2}=\alpha_{2}=p_{2}$ celle bidimensionali di $\Phi$ sono indipendenti. Infatti ogni complesso $A$ costi. tuito da alcune di tali celle, o da tutte, ha il simbolo con i primi $\rho_{z}$ elementi eguali a zero, e i rimanenti non tutti nulli, e pertanto, in base all' ultima osservazione, non può essere anticontorno di un complesso unidimensionale di $\Phi$. Abbiamo così ottenuto $r_{z}$ celle bidimensionali indipendenti, quindi è $\vec{r}_{2} \geq r_{2}$.

Mostriamo ora che è $r_{2} \geq \bar{r}_{2}$, cioè che $r_{2}+1$ celle bidimensionali di $\Phi$, arbitrariamente scelte, sono sempre dipendenti. Ciò equivale a mostrare che, se nel sistema

$$
\begin{aligned}
& \eta_{1,1} x_{1}+\ldots+\eta_{x_{1}, 1} x_{x_{1}}=y_{1} \\
& \ldots \ldots \ldots \\
& \eta_{1, x_{3}} x_{1}+\ldots+\eta_{x_{1}, x_{1}} x_{z_{1}}=y_{x_{3}}
\end{aligned}
$$


si pongono, ad arbitrio, $\alpha_{2}-r_{2}-1=\rho_{2}-1$ delle $y$ eguali a zero, si possono trovare valori non tatti nulli per le rimanenti $y$, tali ohe il sistema (13) venga ad avere soluzioni. Infatti i secondi membri delle (13) vengono in tal caso a costituire il simbolo di un complesso bidimensionale le cui celle sono prese opportunamente entro un gruppo di $r_{z}+1$ celle scelte ad arbitrio.

Supponiamo, per fissare le idee, di porre, nel sistema (13), le prime $\rho_{2}-1$ delle $y$ eguali a zero. Si considerino le prime $\rho_{z}-1$ equazioni di (13), e sia $h$ il massimo numero di equazioni indipendenti che si possono estrarre da esse: supponiamo che siano le prime $h$. Allora si potranno trovare tra le ultime $r_{2}+1$ equazioni $k=\rho_{2}-h$ equazioni che assieme alle prime $h$ formino un sistema di $\rho_{2}$ equazioni i cui primi membri siano indipendenti. Essendo $h \leq \rho_{2}-1$, è $k \geq 1$. Supponiamo che tali equazioni siano quelle situate tra la $\rho_{2}$-esima e la $\left(\rho_{2}+k-1\right)$-esima incluse. Poniamo eguali ad 1 i loro secondi membri. Il sistema (13) diviene allora

$$
\begin{aligned}
& \text { I) }\left\{\begin{array}{l}
\eta_{11} x_{1}+\ldots+\eta_{x_{1}, 1} x_{\alpha_{1}}=0 \\
\ldots \ldots \ldots \\
\eta_{1, h} x_{1}+\ldots+\eta_{x_{1}, h} x_{x_{1}}=0
\end{array}\right. \\
& \text { II) }\left\{\begin{array}{l}
\eta_{1, h+1} x_{1}+\ldots=\eta_{x_{1}, h+1} x_{\alpha_{1}}=0 \\
\cdots \cdots \cdots \cdots \\
\eta_{1, \rho_{2}-1} x_{1}+\ldots+\eta_{x_{1}, \rho_{2}-1} x_{\alpha_{1}}=0
\end{array}\right. \\
& \text { III) }\left\{\begin{array}{l}
\eta_{1 . p_{2}} x_{1}+\ldots+\eta_{\alpha_{1}, p_{2}} x_{\alpha_{1}}=1 \\
\ldots \ldots \ldots \ldots \ldots \\
\eta_{1, p_{2}+k-1} x_{1}+\ldots+\eta_{\alpha_{2}, p_{2}+k-1} x_{\alpha_{1}}=1
\end{array}\right. \\
& \text { IV) }\left\{\begin{array}{l}
\eta_{1, \rho_{2}+\eta_{2}} x_{1}+\ldots+\eta_{\alpha_{1}, \rho_{2}+k_{2}} x_{\alpha_{1}}=y_{p_{2}+k} \\
\cdots \ldots \ldots \\
\eta_{1, x_{2}} x_{1}+\ldots+\eta_{x_{1}, x_{2}} x_{\alpha_{1}}=y_{x_{2}}
\end{array}\right.
\end{aligned}
$$

e risulta diviso in 4 grappi, indicati come I), II), III), IV).

Il primo membro di ciascuna equazione del gruppo IV) è combinazione lineare, secondo certi coefficienti, dei primi membri delle equazioni dei gruppi I) e III) Si combinino linearmente, secondo gli stessi coefficienti, anche i secondi membri, e si diano ai secondi membri delle equazioni del gruppo IV) i valori cosi ottenuti. Allora le equazioni del gruppo II) sono conseguenza di quelle del gruppo I), mentre quelle del grappo IV) sono conseguenza di quelle dei gruppi I) e III), vale a dire ogni soluzione di I) e III) soddisfa anche II) e IV). Ma il sistema formato da I) e III) ammette soluzioni, perchè ha caratteristica eguale al numero delle equazioni; quindi tutto il sistema (13) ammette ora soluzioni. 
Il sistema (13) si è ottenuto dindo ai secondi membri di $\rho_{2}-1$ equazioni arbiturie (che abbiam supposto essere le prime $a_{z}-1$ ) valore zero, e ai secondi membri delle rimanenti, valori opportuni non tutti nulli (perche ¿े $k \geq 1$. Ciò comporta che $r_{2}+1$ celle bidimensionali di $\Phi$, arbitrariamente scelte, son sempre dipendenti. È quindi $r_{z} \geq \bar{r}_{2}$. ML si è già visto essere $\vec{r}_{2} \geq r_{2} ;$ quindi $\vec{r}_{2}=r_{2}$, e. d. d.

9. Il complesso $\Phi$ di cui al n. prec. sia d'ora in poi il complesso topologico, indicato con questa medesima lettera in precedenza, associato al sistema di piani $M$, e alla superficie $S$.

Dato un sistema $H$ di varietà $\Gamma$ su $S$, diremo contomo di H la somma (mod. 2) dei cicli $\Lambda$ contorno della varietà $\Gamma$ di $\mathrm{H}$, vale a dire la somma dei cicli $\Lambda$ ciascun dei quali appartiene a un numero di spazi di varietà $\Gamma$ di $H$.

Più cicli $\Lambda$ si diranno psendodipendenti quando tutti o alcuni tra essi costituiscono il contorno di un sistema di varietc̀ $\Gamma$ su $\mathrm{S}$, mentre si diranno pseudoindipendenti nel caso contrario.

Se più cicli $\mathrm{A}$ di $\mathrm{S}$ costituiscono il contorno di un sistema $\mathrm{H}$ di varietc̀ $\Gamma$ $d i \mathrm{~S}$, a detti cicli $\Lambda$ corrispondono in $\Phi$ celle bidimensionali che costituiscono l'anticontorno del sistema omologo di $\mathrm{H}$ in $\Phi$. La dimostrazione è immediata.

Ne segue che ad ogni sistema di cicli A pseudodipendenti di $\mathrm{S}$ corrisponde un sistema di celle bidimensionali di $\Phi$, e viceversa.

E poichè $r_{2} \dot{\theta}$, come abbiam visto nel n. prec., il massimo numero di celle bidimensionali indipendenti, che si posson trovare su $\Phi$, si ha che anche il massimo numero di cicli A pseudoindipendenti, che si posson trovare su $\mathrm{S}$, eguaglia il secondo rango di connessione $\mathrm{r}_{2} d i \Phi$.

10. D'ora in poi poniamo l'ipotesi restrittiva che la superficie $\mathrm{S}$ sia priva di coefficienti di torsione unidinensionali (e quindi anche bidimensionali) pari.

Ciò comporta (come è noto dalla topologia) che per la $S$ coincidano il rango di connessione e il numero di BETTI di dimensione 1 (e analogamente di dimensione 2).

$\mathrm{Ci}$ occuperemo delle relazioni intercorrenti tra i ranghi di connessione $r_{1}$ ed $r_{2}$ di $\Phi$ e gli invarianti della $S$ (genere geometrico e irregolarità).

Si consideri anzitutto su $\Phi$ un ciclo nnidimensionale $k$, non omologo a zero, che supporremo costituito di un solo circuito. Alle celle zerodimensionali di $k$ corrispondono su $S$ varietà $V$ : siano esse $V_{1}, \ldots, V_{l}$; mentre alle celle unidimensionali di $k$ corrispondono su $S$ varietà $\Gamma$, cjascuna delle quali connette due delle varietà $V_{i}(i=1, \ldots, l)$, Potremo ordinare le $V$ in modo ohe ciascuna di esse sia connessa, mediante una di tali varieta $\Gamma$, alla seguente, e l'ultima alla prima, $\Theta$ indichiamo con $\Gamma_{i, i+1}$ la varietat $\Gamma$ che connette $V_{i}$ con $V_{i+1}(i=1, \ldots, l-1)$, e con $\Gamma_{l, 1}$ quella che connette $V_{l}$ con $V_{t}$. Abbiamo così su $S$ un circuito di varietà $V$ e $T$.

Si fissi, su ciascuna delle $\Gamma_{i, i+1}$ e su $\Gamma_{l, 1}$ un punto $Y_{i, i+1}$, e rispettiva- 
mente $P_{l, 1}$, e congiungiamo $P_{i-1, l}$ con il punto $P_{i, i+1}$ mediante un generico areo di curva $c_{i}$ contenuto in $V_{i}(i=2, \ldots, l-1)$, e analogamente congiungiamo $P_{l-1, l}$ con $P_{l, 1}$, mediante un areo di curva $c_{l}$ in $V_{l}$, e $P_{l, l}$ con $P_{1, \text { ? }}$ mediante un arco $c_{1}$ situato in $V_{1}$. Gli archi $c_{1}, \ldots, c_{\ell}$ costituiscono un circuito $K$ a una dimensione su $\mathrm{S}$.

Mostriamo che il circuito $\mathrm{K}$ non è omologo a zero (mod 2) su S.

Nel seguito di questo numero ei riferiremo sempre ad omologie e congruenze $(\bmod 2)$, anche se ommetteremo di indicarlo ogni volta esplicitemente, per brevità.

Procediamo per assurdo, e supponiamo pertanto che $K$ sia omologo a zero su $S$, vale a dire che esista una varieta bidimensionale $M$ di $S$, avente per contorno $K$.

Pọichè $K$ ha a comune con $\Gamma_{i, i+1}$ il punto $P_{i, i+1}$, la $M$ ha una varietà $I$ a comune con $\Gamma_{i, i+1}$, e tale varietà deve avere dimensione 1 , poichè, se avesse dimensione zero, si potrebbe fare eseguire a $K$ su $M$ un piccolo spostamento in modo che essa non incontri più $\Gamma_{i, i+1}$; e ciò non è. Il contorno di $I$ è formato dall' intersezione di $\Gamma_{i, i+1}$ col contorno di $M$, cioè con $K$, e dall'intersezione di $M$ col contorno di $\Gamma_{i, i+1}$. L'intersezione di $\Gamma_{i, i+1}$ con $K$ è data da $P_{i, i,+1}$; e poichè il contorno di $I$ deve essere un ciclo zerodimensionale, cioè un numero pari di punti, dovrà esistere, oltre $P_{i, i+1}$, un numero dispari di punti che insieme ad esso formano il contorno di $I$ : tali punti si trovano, per quanto si è visto, nel contorno di $\Gamma_{i, i+1}$, vale a dire nei cicli $\Lambda$ situati su $\Gamma_{i, i+1}$. E un namero dispari di tali cicli $\Lambda$ conterrà ciascuno un numero dispari di punti di $I$. Giungiamo quindi, ripetendo il discorso per tutte le varietà $\Gamma_{1,2}, \ldots, \Gamma_{l, 1}$, alla seguente conclusione:

Ciascuna delle varietà $\Gamma_{1,2}, \ldots, \Gamma_{1,1}$ contiene un numero dispari di cicli $A$ che abbiano ciascuno un numero dispari di punti a comune con $\mathrm{H}$.

Consideriamo ora un qualsiasi ciclo $\Lambda$ che abbia un numero dispari di punti a comnne con $M$ : sia esso $\bar{\Lambda}$. Ciascuna delle varietà $\Gamma$ per $\bar{\Lambda}$ deve avere a comune con $M$ un varietà unidimensionale, altrimenti, mediante un piccolo spostamento di $\bar{\Lambda}$ sulla varietà $\Gamma$ considerata, svanirebbero le intersezioni di $\bar{\Lambda}$ con $M$, il che non puô essere, perchè dette intersezioni sono in numero dispari. Sia $\bar{\Gamma}$ una varietà $\Gamma$ per $\bar{\Lambda}$, e sia $J l^{\prime}$ intersezione di $\bar{\Gamma}$ con $M$. Se la $\bar{\Gamma}$ è una delle varietà $\Gamma_{1,2}, \ldots, \Gamma_{l, 1}$, sappiamo gia che su $\bar{\Gamma}$ vi è un numero dispari di cicli $\Lambda$ che abbiano ognuno un numero dispari di punti a comnne con $M$. Supponiamo che invece $\bar{\Gamma}$ non sia una delle varietà $\Gamma_{1,2}, \ldots, \Gamma_{l, 1}$. Allora $\bar{\Gamma}$ non ha punti sul contorno, $K$, di $M$, e di conseguenza il contorno di $J$ è dato unicamente dall'intersezione di $M$ col contorno di $\bar{\Gamma}$, vale a dire dall' inter. sezione di $M$ coi cicli $A$ situati su $\vec{\Gamma}$. E poichè il contorno di $J$ deve essere formato da un numero pari di punti, i cicli $A$ situati su $\bar{\Gamma}$ e aventi un numero dispari di punti a comune con $M$ sono in numero pari. Giungiamo quindi alla conclusione: 
Se un ciclo A ha un numero dispari di punti a comune con М. ogni varietà $\Gamma$ per esso ha una varietà unidimensionale a comune con M. Su ognuna di tali varietà $\Gamma v^{\prime}$ è un numero dispari di cicli $\Lambda$, ciascuno dei quali abbia un numero dispari di punti a comune con $M$, se $\Gamma$ '̀ una delle varietà $\Gamma_{1,2}, \ldots, \Gamma_{1,1}$, mentre v'è un numero pari di tali cicli $\Lambda$, in caso contrario.

Siano $\left.\Lambda^{(}\right), \ldots, \Lambda^{(s)}$ i cicli $\Lambda$ ciascun dei quali abbia un numero dispari di punti a comune con $M$, e siano $\alpha^{(1)}, \ldots, \alpha^{(8)}$ le bicelle di $\Phi$ che corrispondono rispettivamente ai cicli $\Lambda^{(1)}, \ldots, \Lambda^{(s)}$. Siano $\Gamma^{(1)}, \ldots, \Gamma^{(t)}$ le varietà $\Gamma$ contenenti ciaseuna qualcuno dei cicli $\Lambda^{(1)}, \ldots, \Lambda^{(s)}$, e siano $r^{(1)}, \ldots, r^{(t)}$ le 1 -celle di $\Phi$ che corrispondono a $\Gamma^{(1)}, \ldots, \Gamma^{(t)}$. Tra le $r^{(1)}, \ldots, r^{(t)}$ compaiono necessariamente le 1 -celle $r_{1,2}, \ldots, r_{l, 1}$ corrispondenti di $\Gamma_{1,2}, \ldots, \Gamma_{l, 1}$.

In base alla proposizione ultima, possiamo affermare ehe:

Per ogni 1-cella $\mathrm{r}^{(i)}\left(\mathrm{i}=1, \ldots\right.$, t) passa un numero dispari di bicelle $\alpha^{(\mathrm{h})}$, se $\mathrm{r}^{(\mathbf{1})}$ è una delle $\mathrm{r}_{1,2}, \ldots, \mathrm{r}_{1,1}$ mentre passa un numero pari di tali bicelle in caso contrario.

Di qui segue che:

Il complesso bidimensionale $\mathrm{R}$ formato dalle bicelle $\alpha^{(\mathrm{h})}(\mathrm{h}=1, \ldots, \mathrm{s}) e$ delle 1-celle e 0-celle situate sul loro contorno, ha per contorno il circuito unidimensionale formato dalle 1-celle $\mathrm{r}_{1,2}, \ldots, \mathrm{r}_{1,1}$ e dai loro estremi.

$\mathrm{Ma}$ tale cireuito unidimensionale non è che il circuito $k$ di $\Phi$ da cui siamo partiti, e che per ipotesi non è omologo a zero, vale a dire non può essere contorno di un complesso bidimensionale di $\Phi$. Giungiamo quindi ad un assurdo, e per tanto il circuito $\mathrm{K}$ non è omologo a zero.

Concludiamo pertanto:

Se su $\Phi$ si considera un circuito unidimensionale $\mathrm{k}$ non omologo a zero, si ha in corrispondenza ad esso in $\mathrm{S}$ un circuito $\mathrm{X}$ di varietà $\mathrm{V}$ e $\Gamma$, nel quale è contenuto un circuito unidimensionale $\mathrm{K}$ non omologo a zero, incontrante in un punto ciascuna delle varietò $\mathrm{\Gamma}$ di $\mathrm{X}$.

Ripetende il medesimo ragionamento, con minime variazioni, si prova che:

Se su $\mathrm{S}$ si ha un circuito unidimensionale $\overline{\mathrm{K}}$, omologo a $\mathrm{K}$, le 1-celle di $\Phi$ corrispondenti alle varietà $\Gamma$ di $\mathrm{S}$ ciascuna delle quali incontra $\overline{\mathrm{K}}$ in un numero dispari di punti, formano, assieme con le 0-celle ad esse incidenti, un ciclo unidimensionale omologo, $s u \Phi, a \mathrm{k}$.

11. Osserviamo ora quanto segue.

Nel sistema di piani $M$, consideriamo alcune delle rette di connessione, siano esse, $r_{1}, r_{2}, \ldots, r_{n}$, come rette doppie. Otterremo un nuovo sistema di piani $M^{\prime}$. Pensiamo ora una varietà topologica $S^{\prime}$ omogenea e chiusa, la quale abbia per limite $M^{\prime}$, e pertanto si comporti nell' intorno di ogni retta di connessione di $M^{\prime}$ (cioè di ogni retta di $M$ diversa da $r_{1}, \ldots, r_{h}$ ) allo stesso modo in cui si comporta la $S$, ed abbia invece le rette $r_{1}, . ., r_{h}$ come rette doppie. Una tale varietà $S^{\prime}$ potrà ottenersi mediante un opportuno raccordo (previa 
deformazione) degli intorni di $r_{1}, \ldots, r_{h}$ in $M$ con la $S$, cui siano state tolte le varietà $\Gamma$ aventi per limite $r_{1}, \ldots, r_{h}$.

Facciamo ora la seguente

Supposizione $\Sigma$. - La $\mathrm{S}^{\prime}$ abbia, (al pari della $S$ e di ogni riemanniana di superficie algebrica) un numero pari di cicli unidimensionali indipendenti.

Tale supposizione è oltremodo verosimile perchè la $S^{\prime}$ ha, al pari di $S$, come limite un sistema di piani.

1\%. Si consideri ora un circuito unidimensionale $k$ di $\Phi$, non omologo a zero. Ad esso corrisponde su $M$ un circuito di rette e piani che chiameremo $G$. Sia $r_{1}$ una delle rette di connessione di $M$ costituenti $G$, e consideriamo $r_{1}$, anzichè come retta di connessione, come retta doppia: otterremo un nuovo sistema di piani $M_{1}$, cui corrisponde una varieta bidimensionale $\Phi_{1}$, ottenibile da $\Phi$ sopprimendovi la 1-cella corrispondente ad $r_{1}$ e le 2-celle ad essa incidenti. Se $\Phi_{1}$ contiene ancora un circuito $k_{i}$ omologo su $\Phi$ a $k$, si consideri il circuito $C_{1}$ di rette e piani corrispondente di $k_{1}$ su $M_{1}$, e si pensi una sua retta di connessione $r_{2}$ come retta doppia. Si avrà un nuovo sistema di piani $M_{2}$, cui corrisponde la varietà topologica bidimensionale $\Phi_{2}$. Se $\Phi_{2}$ contiene ancora un circuito $k_{\mathrm{g}}$ omologo a $k$ su $\Phi$, si consideri il circuito $C_{2}$ di rette e piani corrispondenti di $k_{2}$ su $M_{2}, \ldots$ e cosl via, sino a che non si giunga ad un sistema di piani $M_{h}$, cui corrisponda una varieta bidimensionale $\Phi_{h}$ priva di circuiti omologhi a $k$ su $\Phi$, e più in generale di cicli omologhi a $k$ su $\Phi$.

Notiamio che il $1^{\circ}$ rango di connessione $d i \Phi_{\mathrm{h}} \grave{e}$ inferiore di una unitò a quello $d i \Phi$. Infatti ei si rende conto facilmente del fatto che ogni ciclo unidimensionale omologo a zero su $\Phi$ che compaia anche in $\Phi_{h}$ è anche ivi omologo a zero, ogni ciclo non omologo a zero su $\Phi$ che compaia anche in $\Phi_{h}$ non è omologo a zero nemmeno su $\Phi_{h}$, e ehe ogni ciclo di $\Phi$ che non compaia più in $\Phi_{h}$ è omologo ad un ciclo di $\Phi_{h}$ più eventualmente il ciclo $k$. Una base pei eicli di $\Phi_{h}$ si può quindi ottenere da una base di $\Phi$ contenente $k$, qualora si sopprima da essa $k$ stesso: donde l'asserto.

Sia ora $S_{h}$ una varietà topologica chiusa e omogenea, che abbia per limite $M_{h}$, costruita al modo indicato al n. 11. Nella $S$, in base al n. 10, e'è un circuito nnidimensionale $K$ giacente nel circuito $X$ di varietà $\Gamma$ e $V$ di $S$ corrispondente a $G$. Inoltre $K$ incontra in un punto ciascuna delle varietà $\Gamma$ giacenti in $X$ e non è omologo a zero su $S$. Quando si passa da. $S$ ad $S_{h}$, il ciclo $K$ svanisce, e, in base all' ultimo enunciato del n. 10, svanisce anche ogni ciclo di $S$ omologo a $K$. Quindi il numero dei cicli unidimensionali indipendenti di $S_{h}$ è inferiore di almeno una unità a quello dei cicli unidimensionali indipendenti di $S$. Ma, in base alla supposizione $\Sigma$, che vale per la $S_{h}$, il numero dei cicli unidimensionali indipendenti di $S_{h}$ è un numero pari, onde il rango di connessione di dimensione $1 d i \mathrm{~S}_{\mathrm{h}} \grave{e}$ inferiore di almeno due unità al rango di connessione di $\mathrm{S}$. 
Se $\Phi_{h}$ possiede cicli nnidimensionali non omologhi a zero, si operi su $S_{h}$, $M_{h}$ e $\Phi_{h}$ come si è operato su $S, M$ e $\Phi$. Si otterranno rispettivamente una varietà quadrimensionale $S_{m}$, un sistema di piani $M_{m}$, e un complesso topologico bidimensionale $\Phi_{m}$. Il rango di connessione di dimensione $1 d i \Phi_{m}$ $\grave{e}$ inferiore di una unità a quello di $\Phi_{\mathrm{h}}$, mentre il rango di connessione di dimensione $1 d i \mathrm{~S}_{\mathrm{m}}$ è inferiore di almeno due unità a quello di $\mathrm{S}_{\mathrm{h}}$.

Se $\Phi_{m}$ contiene cicli unidimensionali non omologhi a zero, si operi su $S_{m}$, $M_{m}$ e $\Phi_{m}$ comé si è operato su $S_{h}, M_{h}$, e $\Phi_{h}$, e così via, sino a che non si giunge ad una varieta bidimensionale $\Phi_{u}$ in oui ogni ciclo unidimensionale è omologo a zero, cui corrisponderà una varietà quadridimensionale $S_{u}$, di rango di connessione positivo o nullo. Ad ogni passo, il rango di connessione di dimensione 1 della varietà bidimensionale diminuisce di una unità, mentre quello della varietà quadrimensionale diminuisce di almeno due unità. Ne viene di conseguenza che il rango di connessione $r_{1}$ della $\Phi$ di dimensione 1 è legato all' analogo rango $R_{1}$ della $S$ dalla relazione

$$
2 r_{1} \leq R_{1}
$$

Ma, in base all'ipotesi posta all'inizio del n. 10, per la $S$ sono eguali il rango di connessione e il numero di BETrI di dimensione 1, e d'altra parte è noto che il numero di Berrr di dimensione 1 di una superficie $S$ è eguale al doppio della sua irregolarità $q$, onde si avrà $2 r_{1} \leq 2 q$, vale a dire

ciò̀:

$$
r_{1} \leq q,
$$

Se la superficie algebrica $\mathrm{S}$ tende al sistema di piani $\mathrm{M}$, l'irregolarità $d i \mathrm{~S}$ non è inferiore al rango di connessione lineare del complesso topologico $\Phi$ associato ad M, purchè la $S$ non contenga cicli lineari pseudonulli di periodo pari. 